\title{
Participants Ranking Algorithm for Crowdsensing in Mobile Communication
}

\author{
Tariq Ali ${ }^{1}$, Javeria Noureen, Umar Draz, Ahmad Shaf, Sana Yasin, and Muhammad Ayaz ${ }^{2}$ \\ 1 Computer Science Department, CIIT, Sahiwal, Pakistan, tariqali@ciitsahiwal.edu.pk \\ 2 Sensor Networks and Cellular Systems (SNCS) Research Centre, University of Tabuk, Tabuk, KSA
}

\begin{abstract}

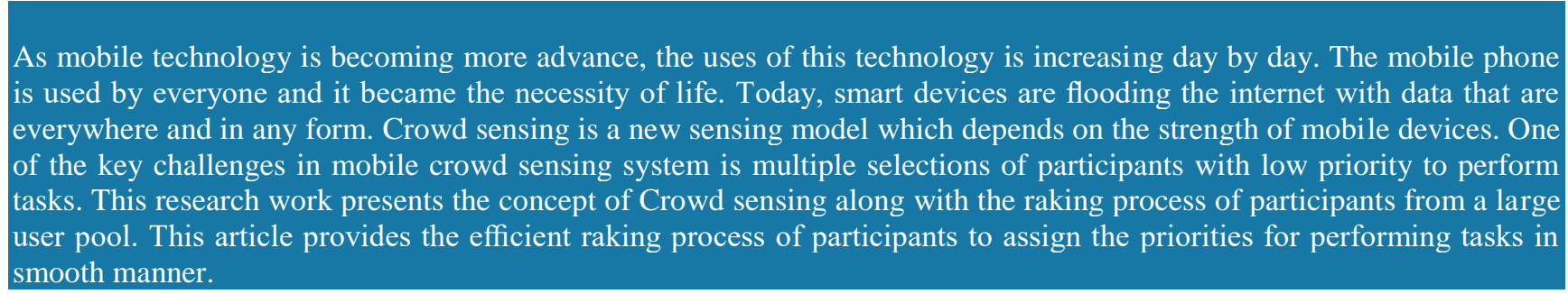

Keywords: Crowdsensing, Participant, Crowd Sourcing, Recruitments, User Pool.

Received on 23 November 2017, accepted on 10 February 2018, published on 13 April 2018

Copyright (C) 2018 Tariq Ali et al., licensed to EAI. This is an open access article distributed under the terms of the Creative Commons Attribution licence (http://creativecommons.org/licenses/by/3.0/), which permits unlimited use, distribution and reproduction in any medium so long as the original work is properly cited.

doi: 10.4108/eai.13-4-2018.154476

\section{Introduction}

Today mobile phone is the essential part of life. As the data for different scientific studies. For example, the human volunteers help the Great Backyard Bird Count years passing, the use of mobile phones has greatly increased; the latest mobile phones now come with many embedded sensors. The capabilities of mobile phones have been greatly increased in the recent years, for instance processing power, embedded sensors, storage capacities and network information rates. These advances technologies combined with the vast number of client companioned cell phones empower another and quickly developing sensing paradigm called Crowd sensing. Crowd sensing is the ability by which application developers can make tasks and recruit cell phone clients to give sensor information to be utilized towards a design goal. Crowd sensing is also sometimes referred as a mobile Crowd sensing. A formal way to represent the MCS (mobile Crowd sensing) is: Mobile Crowd Sensing (MCS) presents a new sensing model, which is based on the power of mobile devices. The absolute number of user companioned devices such as mobile phones, wearable devices and smart vehicles so on $[1,2]$ and their inherent mobility empowers a new and fast-growing sensing paradigm that empowers ordinary citizens to contribute data sensed or generated from their mobile devices, aggregates and fuses the data in the cloud for crowd intelligence extraction and people-centric service delivery [2, 3]. Mobile crowd sensing (MCS) permits a huge amount of mobile phone users that share local knowledge (e.g., local information, ambient context, noise level, and traffic conditions) collected by their sensor-enhanced devices. According to mobile phone use for computation and acquires a richer functionality. It has a variety of sensors such as camera, microphone, Global Positioning System, accelerometer, digital compass, light sensor, Bluetooth as proximity sensor. Health and pollution monitoring sensors will be intended in the coming future. Mobile sensors such as smart phones and vehicular systems represent a new type of geographically distributed sensing infrastructure that enables mobile people-centric sensing. Until recently mobile sensing research such as activity recognition, where peoples activity (e.g., walking, driving, sitting, talking) is 
classified and monitored, required specialized mobile devices (e.g., the Mobile Sensing Platform [MSP] [4]. Crowdsensing have different applications which are divided into three categories:

1. Infrastructure monitoring

2. Social networking monitoring

3. Environmental monitoring [5]

\section{Crowdsensing Challenges}

Crowd sensing has many challenges here we discussed some privacy and security challenges [6] and limitation for mobile crowd sensing with the viable solutions. Local analytics is key challenge in discovering heuristics and designing algorithms is to complete the imaginary function. Data mediation is one of the classes of functions, for example clarifying of outliers, noise exclusion, or covering data gaps. For instance, GPS sample cannot be able to obtain correct or missing (due to absence of line of sight), in this event outliers must be eliminating or omitted samples extrapolated. The 3-tier system architecture [13] also have some challenges are as follows: (a) Virtualization Overhead is the main challenge in system architecture. (b) Configuration and performance is another challenge of inter-VM communication. (c) Inter-VM communication performance is comparatively low when it is compare to inter-process communication. Migration-induced Reconfiguration is likewise challenges. The most suitable possible solution is: With constraints of Non-IP-based solutions, the Host Identify Protocol [7] are designed to scratch mind, still these protocols are essential for the evaluation of real networks. Different crowd sensing applications can construct similar sensor data, but use diverse system or model rate. Another challenge is how to provide valuable incentive mechanisms that allows honest contributions in mobile crowd sensing and computing becomes a critical challenge [3]. The solution is recently, numerous game theory approaches [8] have been proposed for mobile crowd sensing and computing to encourage and reward truthful contributions. For a highly dynamic mobile crowd sensing and computing system, there is still need for new incentive and pricing mechanisms to attract, inspire, and reward truthful and high-quality sensing data contributors. For data delivery Data delivery in transient network is also challenge in mobile crowd sensing, how to dispatch the sensed data from distributed participants to the backend server is another challenge because of an assortment of mobile crowd sensing and computing characteristics, for example the low bandwidth of wireless communication, recurrent network apportioning due to human mobility, and vast number of energy-constrained devices. Scheduling algorithms can solve this trouble and used sensing servers to arrange sensing events of mobile phones (an incentive mechanism use recruited). Note that opportunistic sensing applications will only use the scheduling algorithms; later, in participatory sensing applications; mobile phone users control sensing task by manually [9].

\subsection{Privacy in Crowdsensing}

Privacy is very important for everyone. No one wants to reveal his /her privacy in front of anyone. We can use different techniques to provide privacy to mobile devices or nodes. Data collection infrastructure layer is used to collect data from the selected sensor nodes. It provides privacy-preserving mechanisms for data contributors. Some component such as task allocation, sensor gateways, data anonymization, incentive mechanism and big data storage are used in this layer, which collected data from the selected nodes [3]. With the wide adoption of mobile Crowd sensing applications, task coverage and participant selection in MCS system has capture the attentions of researchers. First, there are several system and experimental studies on either experimental study on MCS coverage or general framework of participant recruitment. For example, in has performed a systematic study of the coverage and scaling properties of placecentric urban crowd sensing and shows promising results that MCS can provide relatively high coverage levels especially given area with large size. Then, there are also many theoretical studies on various task assignment and participant selection problems, playing tradeoffs among sensing cost, task coverage, energy efficiency and user privacy, and incentive [10, 11]. In study [9] offline participant selection in piggyback MCS for probabilistic coverage. They aim to select minimum number of participants to guarantee the selected participants will make enough number of calls at certain percentage of the target locations over a long-fixed sensing period. There is some privacy and privacy challenges or risk where campaign administrator breaks the trust among participants and reveals the sensitive data about participants. We will focus on the social and technical challenges or threats. Fig. 1 is reference by [12]. In this paper, different concept are presents such as Crowd sensing, Crowd sensing application, Crowd sensing challenges and privacy issues and Crowd sensing related work. Mobile Crowd sensing (MCS) permits the large amount of cell phone clients share native knowledge such as (local information, ambient

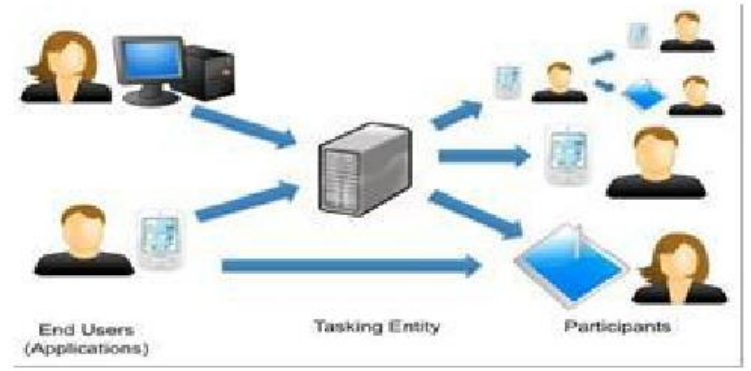

Fig. 1. Generic structure of task flow in MCS

context, noise level, and traffic conditions) collected by their sensor-enhanced devices, and more information can be collects in the cloud for large scale sensing and community intelligence mining [15]. Crowd sensing has 
many challenges some privacy and security challenges $[13,14]$ are mentioned. To get the privacy different privacy techniques can use to protect the user privacy such as Anonymization, encryption and data perturbation but still there is some privacy threats need to be solved. The key challenge which identify in this research work is how to effectively identify the well-suited participant in the large user pool. The recruitment process becomes more complicated when the sensing tasks are dynamic and heterogeneous.

\section{Methodology}

The ranking algorithm ranked the participants from the large user pool. Ranking assigns on the base of participant availability and then selects the top ranked participants to assign task. Suppose there are ten participants in the user pool, based on the participant availability in user pool, the ranking is assigned. The top ranked participants to assign a task. So, in the table 1 , we can see that user $1,2,3,7,8$ and 9 has top ranking. Out of these top ranked participants will perform tasks they have highest priority. Table 1 shows the ranking of participants.

Table 1. Participants Raking

\begin{tabular}{|c|l|l|l|l|l|l|l|l|l|l|}
\hline Users & 1 & 2 & 3 & 4 & 5 & 6 & 7 & 8 & 9 & 10 \\
\hline Ranking & 2 & 5 & 3 & 1 & 2 & 1 & 5 & 4 & 3 & 1 \\
\hline Ranked Participants & & $\mathrm{S}$ & $\mathrm{S}$ & & & & $\mathrm{S}$ & $\mathrm{S}$ & $\mathrm{S}$ & \\
\hline
\end{tabular}

In the ranking algorithm select the user from user pool based the top raking. Top ranking provides because of participants availability.Table 2 shows the ranking algorithm for selection of the participants.

Table 2. Raking algorithm

\begin{tabular}{|c|c|}
\hline Users & $\begin{array}{r}\text { We have N number of users which are taking part } \\
\text { in user pool. Users }=1,2,3,, \mathrm{~N} .\end{array}$ \\
\hline Ranking & $\begin{array}{c}\text { Participants are selected through ranking. Ranking }=1,2,3,, 5 . \\
\mathrm{X}\end{array}$ \\
\hline
\end{tabular}

\subsection{Pseudocode of Raking Algorithm}

Input: 1 to $\mathrm{N}$ numbers of participants Output: ranked participants

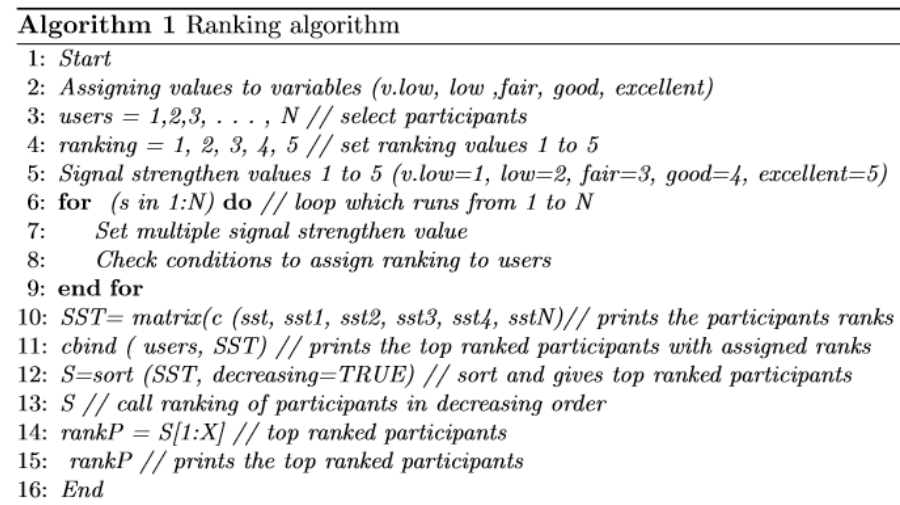

\subsection{Working of Raking Algorithm}

In Fig. 2, the working of raking algorithm is show, the raking of mobile $\mathrm{N}$ number of user is based on the value of signal strength. The user will get highest ranking if it has maximum value out five. The five is assuming value for sorting the raking of users from the large pools.

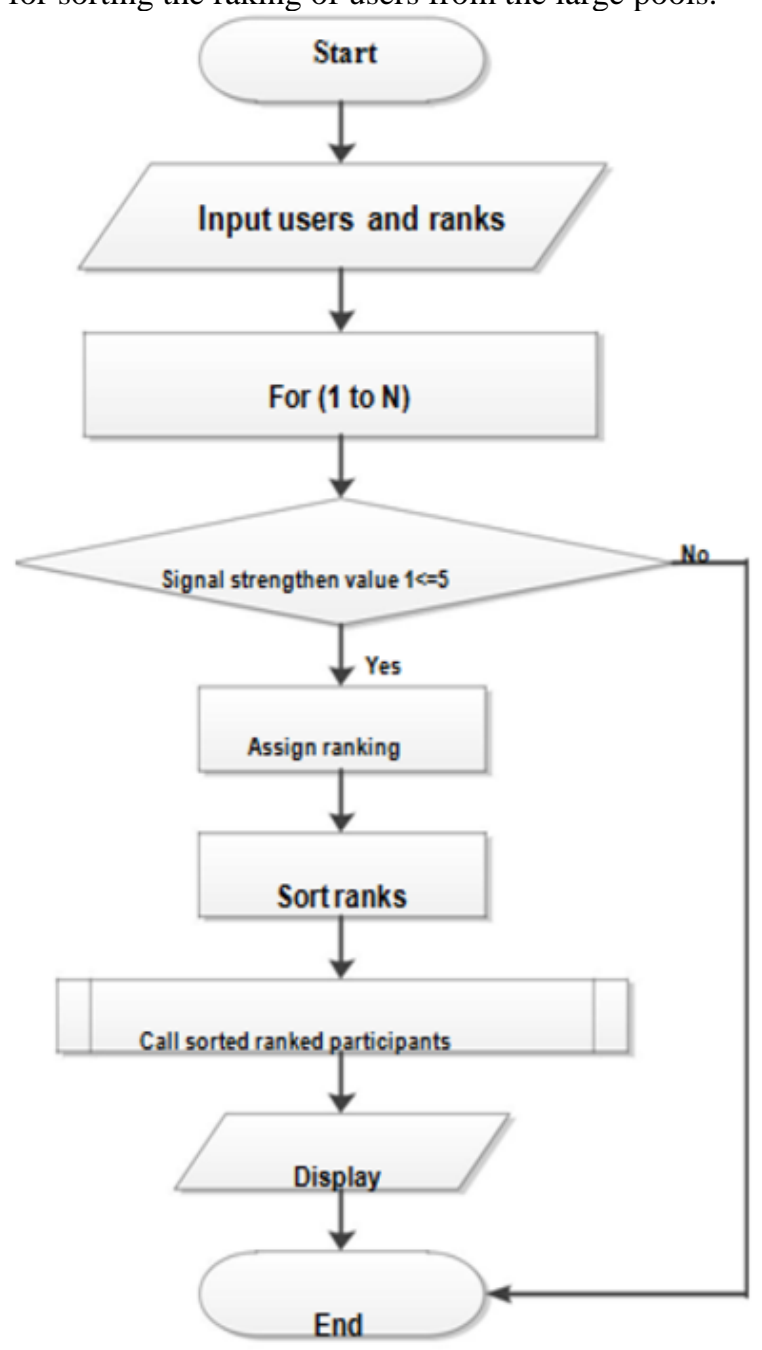

Fig. 2. Flow chart for raking algorithm 


\section{Results and Discussion}

In the Ranking of $\mathrm{N}$ numbers of Participants Recruitment Algorithm, there are three different tasks such as Task1, Task2 and Task3. For these three tasks, ranking algorithm ranks the participants. Top rank participants will be selected in each task. There are some results of the participants ranking for three different tasks are taken but Task 1 is explained as an example. In the Task1, primarily it is assumed that there are 10 numbers of participants and these participants have different ranking which relay 1 to 5. Every participant has different ranking on the base of mobile signal strength. Only those five participants will be selected which has top ranking. Fig. 3 shows the generic code of participants ranking algorithm while Fig. 4 shows the result of participant ranking algorithm for Task1.

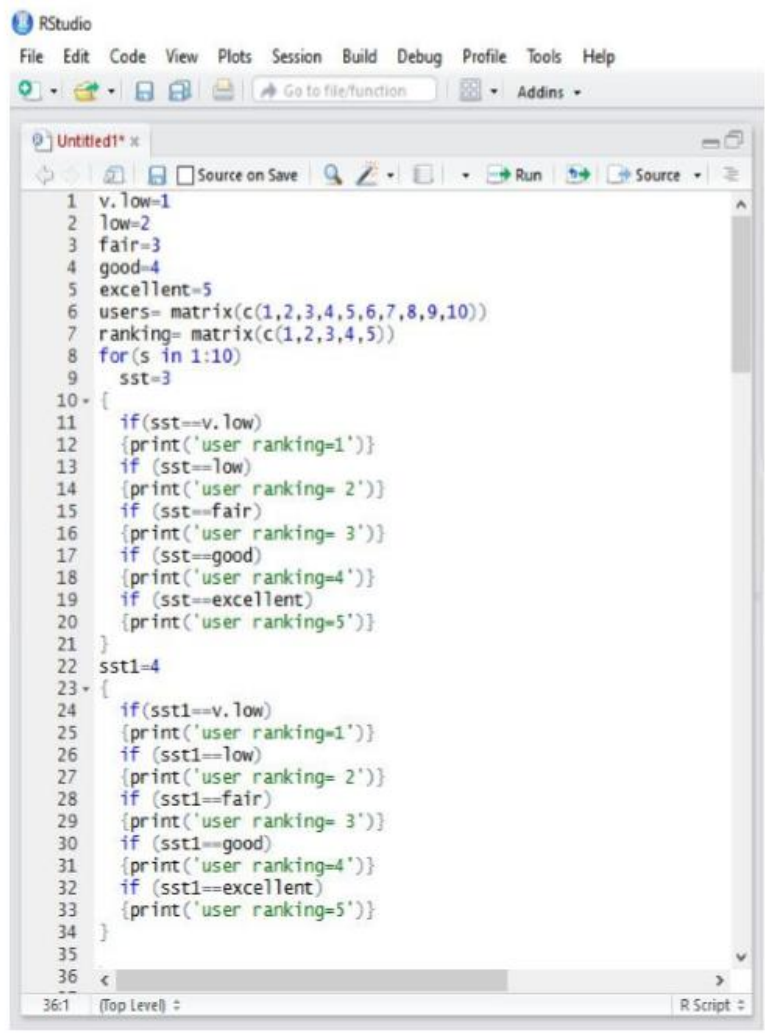

Fig. 3. Participants Ranking Code for Tasks

It is assumed that participants have different ranking on the bases of signal strength. Fig. 4 shows the participants ranking algorithm result for Task 1 are as follows:

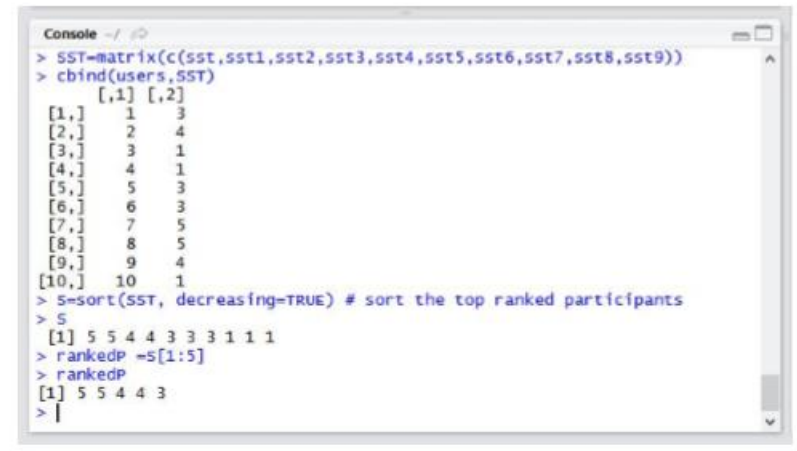

Fig. 4. Participants Ranking Result for Task1

Table 3 shows that 10 users have different ranking. Ranking algorithm dynamically assign the ranking to the participants on the base of signal strength. After assigning different ranking to the user, algorithm sort the top ranked users and then choose the first top 5 users, to assign task. In the following table, $\mathrm{S}$ denoted the top users whom are selected to assign tasks. User $1,2,7,8$ and 9 are the top ranked users for assigning task.

Table 3. Participants Ranking for Task1

\begin{tabular}{|c|l|l|l|l|l|l|l|l|l|l|}
\hline Users & 1 & 2 & 3 & 4 & 5 & 6 & 7 & 8 & 9 & 10 \\
\hline Ranking & 3 & 4 & 1 & 1 & 3 & 3 & 5 & 5 & 4 & 1 \\
\hline Ranked Participants & $\mathrm{S}$ & $\mathrm{S}$ & & & & & $\mathrm{S}$ & $\mathrm{S}$ & $\mathrm{S}$ & \\
\hline
\end{tabular}

\subsection{Graphical Representation of Results}

Fig. 5 shows ranking of the participants for three different tasks. The top five ranking participants are selected to assign task. The values of table 3 have been used to evaluate multiple tasks for graphical presentation of results.

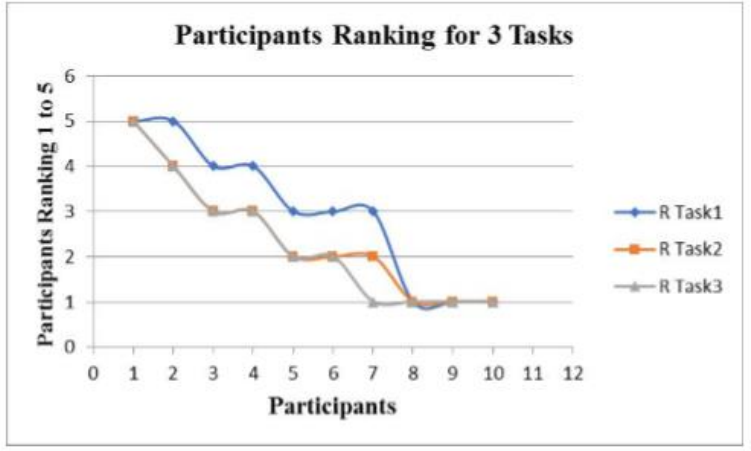

Fig. 5. Participants ranking for Tasks

\section{Conclusion}

Literature is reviewed to understand the dimensions related to ongoing and emerging issues in mobile crowd 
sensing. Different studies are analyzed to identify the focus of studies such as mobile crowd sensing, its applications domains, privacy and security challenges and limitation for mobile crowd sensing with their possible solution. Then existing participant raking process is analyzed. We have identified few shortcomings in the mobile crowd sensing system is how to effectively be ranked the well-suited participants in recruitments from a large user pool and suggested few algorithms that can improve the efficiency of algorithm. The recommendations presented for the existing participant recruitment process that can improve the recruitment process, how we can recruit the wellsuited participants from the large user pool. In this paper, the raking algorithm is proposed to assign the priority to multiple users. This algorithm efficiently ranks the participants and improves the performance of algorithm. The Selection of $\mathrm{N}$ number of participant recruitment algorithm, with goal to minimizing the sensing cost while satisfying the certain level of coverage. In future, we are interested to extend this research by developing energy efficient sensor device that will minimize the energy consumption and will increase the battery life of the smart phones.

\section{References}

1. Du, R., et al. Predicting activity attendance in eventbased social networks: Content, context and social influence. in Proceedings of the 2014 ACM International Joint Conference on Pervasive and Ubiquitous Computing. 2014. ACM.

2. Guo, B., et al. From participatory sensing to mobile crowd sensing. in Pervasive Computing and Communications Workshops (PERCOM Workshops), 2014 IEEE International Conference on. 2014. IEEE. 10 Tariq Ali et al.

3. Guo, B., et al., Mobile crowd sensing and computing: The review of an emerging human-powered sensing paradigm. ACM Computing Surveys (CSUR), 2015. 48(1): p. 7.

4. Lane, N.D., et al., A survey of mobile phone sensing. Communications Magazine, IEEE, 2010. 48(9): p. 140150.

5. Dimov, D., Crowdsensing: State of the Art and Privacy Aspects. InfoSec Institute, 2014. 29.

6. Maisonneuve, N., M. Stevens, and B. Ochab, Participatory noise pollution monitoring using mobile phones. Information Polity, 2010. 15(1, 2): p. 51-71.

7. Rana, R.K., et al. Ear-phone: an end-to-end participatory urban noise mapping system. in Proceedings of the 9th ACM/IEEE International Conference on Information Processing in Sensor Networks. 2010. ACM. 8. Azizyan, M., I. Constandache, and R. Roy Choudhury. SurroundSense: mobile phone localization via ambience fingerprinting. in Proceedings of the 15th annual international conference on Mobile computing and networking. 2009. ACM.
9. Lu, H., et al. SoundSense: scalable sound sensing for people-centric applications on mobile phones. in Proceedings of the 7th international conference on Mobile systems, applications, and services. 2009. ACM. 10. Narula, P., et al., MobileWorks: A Mobile Crowdsourcing Platform for Workers at the Bottom of the Pyramid. Human Computation, 2011. 11: p. 11.

11. Mardenfeld, S., et al. Gdc: Group discovery using colocation traces. in Social computing (SocialCom), 2010 IEEE second international conference on. 2010. IEEE. 12. Ganti, R.K., F. Ye, and H. Lei, Mobile crowdsensing: current state and future challenges. Communications Magazine, IEEE, 2011. 49(11): p. 32-39.

13. Heggen, S., A. Adagale, and J. Payton, Lowering the barrier for crowdsensing application development, in Mobile Computing, Applications, and Services. 2013, Springer. p. 1-18.

14. Nikander, P., A. Gurtov, and T.R. Henderson, Host identity protocol (HIP): Connectivity, mobility, multihoming, security, and privacy over IPv4 and IPv6 networks. Communications Surveys \& Tutorials, IEEE, 2010. 12(2): p. 186-204.

15. Huangfu, S., et al. Using the model of markets with intermediaries as an incentive scheme for opportunistic social networks. in Ubiquitous Intelligence and Computing, 2013 IEEE 10th International Conference on and 10th International Conference on Autonomic and Trusted Computing (UIC/ATC). 2013. IEEE. 